2 Riccardo Montis ${ }^{1,7 *}$, Luca Fusaro ${ }^{2}$, Andrea Falqui ${ }^{3}$, Michael B. Hursthouse ${ }^{1}$, Nikolay Tumanov²,

3 Simon J. Coles ${ }^{1}$, Terry L. Threlfall ${ }^{1}$, Peter N. Horton ${ }^{1}$, Rachid Sougrat ${ }^{4}$, Anaïs Lafontaine ${ }^{5}$, Gérard

4 Coquerel $^{5}$ and A. David Rae ${ }^{6}$

$5{ }^{1}$ School of Chemistry, University of Southampton, Highfield, Southampton SO17 1BJ, UK.,

$6 \quad{ }^{2}$ Namur Institute of Structured Matter (NISM) University of Namur, Namur, Belgium, ${ }^{3}$ King

7 Abdullah University of Science and Technology (KAUST), Biological and Environmental

8 Sciences and Engineering (BESE) Division, NABLA Lab, 23955-6900 Thuwal, Saudi Arabia,

$9 \quad{ }^{4}$ King Abdullah University of Science and Technology (KAUST), KAUST Core Labs, 23955-

106900 Thuwal, Saudi Arabia, ${ }^{5}$ Laboratoire SMS-EA3233, Université de Rouen Normandie, F-

1176821 Mont Saint Aignan, France, ${ }^{6}$ Research School of Chemistry, College of Physical Sciences,

12 Australian National University, Canberra ACT 0200, Australia, ${ }^{7}$ Current address: Department of

13 Chemical Engineering and Analytical Science, The University of Manchester, The Mill, Sackville

14 Street, Manchester M1 3AL, UK.

Molecular self-assembly is the spontaneous association of simple molecules into larger and ordered structures. ${ }^{1}$ It is the basis of several natural processes, such as the formation of colloids, crystals, the generation of proteins, viruses and double helical DNA. ${ }^{2}$ Molecular selfassembly has inspired strategies for the rational design of materials with specific chemical/physical properties, ${ }^{3}$ becoming perhaps one of the most fascinating and important concepts in supramolecular chemistry. Here we report on a simple hydrochloride salt of fampridine which crystallises as four different structures, two of which adopt unusual selfassemblies consisting of polyhedral clusters of chloride and pyridinium ions. These two 
structures represent the first observation of Frank Kasper (FK) phases of a small and rigid organic molecule. Although discovered in metal alloys ${ }^{4-5}$ more than 60 years ago, FK phases have recently been observed in several classes of supramolecular soft matter ${ }^{6-11}$ and in gold nanocrystal superlattices ${ }^{12}$ and still are the object of new discoveries. ${ }^{13}$ In these systems, atoms or spherical assemblies of molecules are packed to form polyhedra with coordination numbers (CN) 12, 14, 15 or 16. The two FK structures reported here crystallise from a dense liquid phase and show a complexity that is generally not observed in the case of rigid and small organic molecules. Investigation of the precursor dense liquid phase by cryo-EM reveals the presence of spherical aggregates with size ranging between 1.5 and $5 \mathrm{~nm}$. These structures, together with the experimental procedure used for their preparation, invite interesting considerations on the route to their formation and open new perspectives for the design of a new generation of organic crystalline materials.

Results from previous crystallographic studies on salts of fampridine, ${ }^{14-17}$ a voltage-dependent potassium channel blocker used for the treatment of multiple sclerosis, occasionally showed unexpected self-assemblies of the components but none of the complexity mentioned above. Conversely, the new structures presented here have complex and unique features, including one of the largest numbers to date of molecules in the asymmetric unit $\left(Z^{\prime}=30\right)$.

The four phases of fampridine hydrochloride $\left(4-\mathrm{APH}^{+} \mathrm{Cl}^{-}\right)$, comprise two analogous sub-hydrate forms 4- $\mathrm{APH}^{+} \mathrm{Cl}^{-} 1 / 12 \mathrm{H}_{2} \mathrm{O}$ (phase 1) and 4- $\mathrm{APH}^{+} \mathrm{Cl}^{-} 1 / 90 \mathrm{H}_{2} \mathrm{O}$ (phase 2), a monohydrate, 4$\mathrm{APH}^{+} \mathrm{Cl}^{-} \mathrm{H}_{2} \mathrm{O}$ (phase 3), and an anhydrous form, 4- $\mathrm{APH}^{+} \mathrm{Cl}^{-}$(phase 4).

Phase 1 was prepared by several procedures, the consistency of which has been confirmed by reproduction in three separate laboratories. In most cases, crystals of phase $\mathbf{1}$ were obtained directly by mixing equimolar amount of 4-AP and concentrated $\mathrm{HCl}$ into solutions of ethanol or methanol 
1 followed by acetone diffusion into the mixture. Interestingly, this generated a liquid-liquid phase

2 separation (LLPS) consisting of a solvent rich phase and a dense liquid phase (DLP), from which

3 prism/block-like colourless crystals were obtained (Extended Data 1). Phase 1 proved to be stable

4 if kept in reasonably anhydrous conditions. Single-crystal X-ray investigations were made on

5 several crystals, from different preparations, resulting in the same, partly disordered phase $\mathbf{1}$

6 structure in the space group $C 2 / c$, with occupancies that are multiples of $1 / 6$ and an asymmetric

7 unit consisting of 30 independent 4- $\mathrm{APH}^{+} \mathrm{Cl}^{-}$and 2.5 molecules of $\mathrm{H}_{2} \mathrm{O}$. Phase $\mathbf{1}$ can be described

8 as a modulation of a parent structure of $P m 3 n$ space group symmetry with an axial length of about

$927.5 \AA$ (Figure 1a). Attempts to recrystallize phase $\mathbf{1}$ from the melt produced single crystals of

10 phase 2. Occasionally, from the DLP separated during the preparation of phase $\mathbf{1}$, some crystals of

11 phase $\mathbf{2}$ appeared concomitantly. Phase $\mathbf{2}$ crystallises in the tetragonal crystal system (space group

$12 P 4_{2} / m$ ) with $Z^{\prime}=15$ and shows subtle structural differences when compared to phase $\mathbf{1}$ (Figure $1 \mathrm{~b}$ ),

13 as well as a lower water content ( $1 / 90$ for each $\left.4-\mathrm{APH}^{+} \mathrm{Cl}^{-}\right)$.

14 When viewed along the crystallographic $c$ axis, phases $\mathbf{1}$ and $\mathbf{2}$ are seen to contain three types of

15 cluster species (A, B and C) with sizes in the range of 1.2-1.5 nm. These clusters are comprised of

16 polyhedral assemblies of $\mathrm{Cl}^{-}$and $4-\mathrm{APH}^{+}$, with cations lying at the centre of hexagons and

17 pentagons and interacting with surrounding $\mathrm{Cl}^{-}$anions via sets of $\mathrm{N}-\mathrm{H} . . . \mathrm{Cl}^{-}$and $\mathrm{C}-\mathrm{H} . . . \mathrm{Cl}^{-}$contacts

18 (Figure 2a). There are two instances of clusters A (A1 and A2), mainly differing for the orientation

19 of $4-\mathrm{APH}^{+}$ions and the symmetries relating the $4-\mathrm{APH}^{+} \mathrm{Cl}^{-}$building units. Both consist of four

20 cations surrounded by a pattern of sixteen chlorides to form a bicapped hexagonal antiprism, also

21 known as the Friauf polyhedron (Figure 2b, purple and orange). Cluster B (Figure 2b, blue)

22 consists of a pair of cations lying at the centre of an icosahedron of twelve chlorides. In both 
1 phases, water molecules are positioned in regions between clusters A and B, interacting with $\mathrm{Cl}^{-}$

2 via $\mathrm{O}-\mathrm{H} . . . \mathrm{Cl}$ hydrogen bonds.

3 FIGURE 1 here

4 In both structures $\mathbf{1}$ and 2, clusters A and B are built using all the symmetrically independent 5 chlorides (30 and 15 respectively), but only a portion of the symmetrically independent 4- $\mathrm{APH}^{+}$

6 cations (7 and 5 respectively). The remaining 4- $\mathrm{APH}^{+}$cations (23 and 10 for phase $\mathbf{1}$ and $\mathbf{2}$

7 respectively) occupy interstitial sites, resulting in the formation of C clusters (Figure 2b, grey).

8 Clusters $\mathrm{C}$ consist of four cations surrounded by a pattern of twelve chlorides to form a truncated

9 tetrahedron, also known as a Laves polyhedron. Clusters A, B and C represent an unprecedented

10 self-assembly of small organic molecules, reminiscent of patterns observed in water clathrates. ${ }^{18}$

\section{FIGURE2 here}

12 The crystal packing of $\mathbf{1}$ and $\mathbf{2}$ consists of infinite columns of A1 and A2 (Figure 2c) separated by

13 isolated instances of B, all connected via interstitial C clusters. In the two structures, the clusters

14 adopt slightly different orientations, generating two virtually identical crystal packings (Figure 1).

15 Each A cluster, is coordinated to four B clusters and to a total of $10 \mathrm{~A}$ clusters $(\mathrm{CN}=14)$, while $\mathrm{B}$ is

16 coordinated to a total of twelve A clusters $(\mathrm{CN}=12)$. In both structures, $\mathrm{A}$ and $\mathrm{B}$ are found in a 3:1

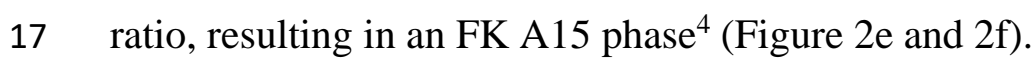

18 To the best of our knowledge, phases $\mathbf{1}$ and $\mathbf{2}$ are the first observation of FK crystal structures of a

19 small, rigid organic molecule, representing a missing piece in the complex scenario of FK phases.

20 On the contrary, phase 3 adopts a simpler crystal packing, similar to those previously reported for

21 salts of 4-AP. ${ }^{14-17}$ Phase 3 was crystallised by evaporation from aqueous solutions, resulting in a 
1 triclinic unit cell with Z' = 4 (Extended Data 2). Phase 4 adopts a relatively simple crystal packing;

2 however, it shows hexagonal patterns reminiscent of those observed in polyhedral clusters of phases

$3 \mathbf{1}$ and 2, but in this case arranged as 2-D planes (see section 3 in methods). So far, only phases $\mathbf{1}$ and

43 could be reproducibly crystallised from solutions, while phases 2 and 4 have been mainly obtained

5 from the melt.

6 When we compare phases $\mathbf{1}$ and $\mathbf{3}$ and their condition of crystallisation, it is pertinent to question

7 how is it possible that the same building unit can nucleate and grow, forming such different crystal

8 structures. Considering the complexity of $\mathbf{1}$, it seems highly improbable that simple $4-\mathrm{APH}^{+} \mathrm{Cl}^{-}$

9 entities could reproducibly come together molecule-by-molecule to form such a complex crystal

10 structure. Therefore, we believe that some specific pre-organisation must be in place prior to

11 crystallisation of $\mathbf{1}$, promoting its nucleation and growth. In contrast to the simple phase $\mathbf{3}$,

12 crystallisation of phase $\mathbf{1}$ occurs via a DLP-mediated process, involving a preliminary LLPS. We

13 therefore monitored the crystallisation of phases $\mathbf{1}$ and $\mathbf{3}$ by liquid-state NMR, with the aim to

14 identify any difference in the composition, intermolecular interactions and aggregation between the

15 DLP precursor of phase $\mathbf{1}$ and the aqueous solution precursor of the simple phase 3 . In these two

16 crystallisation experiments, hereafter named as $\mathbf{L} \mathbf{1}$ (precursor of phase $\mathbf{1}$ ) and $\mathbf{L} \mathbf{3}$ (precursor of phase

17 3), the liquids were monitored by ${ }^{1} \mathrm{H}$ NMR and ${ }^{35} \mathrm{Cl}$ NMR as a function of the concentration of 4-

$18 \mathrm{APH}^{+} \mathrm{Cl}^{-}$until the moment when precipitation of the crystalline phases $\mathbf{1}$ and $\mathbf{3}$ occurred (Figure 3a,

19 3b and Supplementary Information). The results show strong differences between the two liquids,

20 in particular near the crystallization point. Phase $\mathbf{1}$ crystallised when $\mathbf{L} \mathbf{1}$ contained approximately

211 equivalent of water, 0.8 equivalents of acetone and small amounts of ethanol (approximately 0.1

22 equivalents), while phase $\mathbf{3}$ crystallised from $\mathbf{L} 3$ in the presence of 2 equivalents of water. These

23 differences in the composition are also reflected in the chemical shift variation of ${ }^{1} \mathrm{H}$ and ${ }^{35} \mathrm{Cl}$ as the 
1 function of the increasing molar fraction of $4-\mathrm{APH}^{+} \mathrm{Cl}^{-}$(Figure 3c and 3d). In both cases all the

2 signals upshifted, suggesting self-association of 4- $\mathrm{APH}^{+} \mathrm{Cl}^{-}$. However, the chemical shifts of $\mathbf{L 1}$

3 were generally higher when compared to those observed for $\mathbf{L} \mathbf{3}$, suggesting that the presence of

4 acetone and ethanol in $\mathbf{~} \mathbf{1}$ influences the chemical environment of $4-\mathrm{APH}^{+} \mathrm{Cl}^{-}$, due to different

5 solvent-solute interactions and, consequently, a different self-assembly.

6 The spin-lattice relaxation times $\left(\mathrm{T}_{1}\right)$ of the aromatic $\mathrm{CH}$ for $\mathbf{L} \mathbf{1}$ and $\mathbf{L} \mathbf{3}$ were also measured (see

7 Supplementary Information). In both cases, $\mathrm{T}_{1}$ decreased with increasing concentrations of 4-

$8 \mathrm{APH}^{+}$, eventually converging to a similar value just prior the crystallisation of phase $\mathbf{1}$ and $\mathbf{3}$. This

9 was ascribed to a close rotational correlation time and, consequently, to a similar viscosity of $\mathbf{L 1}$

10 and $\mathbf{L} 3$ at high concentrations.

11 In order to investigate the dynamics of the ionic species, the full linewidth at half height (LW) of

12 the signals of quadrupolar ${ }^{14} \mathrm{~N}$ and ${ }^{35} \mathrm{Cl}$ nuclei was also measured. This strongly depends on the

13 viscosity of the medium and the symmetry of the species. Interestingly, the $\mathrm{LW}$ values of the ${ }^{35} \mathrm{Cl}$

14 nucleus for $\mathbf{L} \mathbf{1}$ are consistently higher when compared to those of $\mathbf{L 3}$ (Figure 3e). Considering the

15 similar viscosity of $\mathbf{L} \mathbf{1}$ and $\mathbf{L 3}$ at high concentrations, the differences observed for the LW of the

$16 \quad{ }^{35} \mathrm{Cl}$ nucleus must be ascribed to a different self-assembly, prior the nucleation of phases $\mathbf{1}$ and 3.

17 FIGURE 3 here

18 In order to probe the microstructure of L1 prior the crystallization of phase 1, Cryo-EM was

19 performed. Due to the very pronounced tendency of the sample to sublime under the electron beam,

20 we worked upon low electron dose conditions, resulting in images with low signal to noise ratio and

21 limited resolution. The Cryo-EM results suggest the presence of small spherical objects and different

22 types of aggregates, with sizes ranging between 1.5 - $4.6( \pm 0.5) \mathrm{nm}$ (see Figure 3f and Extended 
1 Data 7). These objects show a size and a shape that is consistent with clusters and aggregates

2 observed in crystal structures of $\mathbf{1}$ and 2, supporting the idea that the complexity of their crystal

3 structures arises from a pre-organisation in the liquid state.

4 Our results indicate that the formation of the DLP have an important role on the crystallisation of

5 the complex phase 1 and presumably of phase 2. LLPS prior to crystal formation has been previously

6 documented in several protein systems ${ }^{19}$ and more recently ${ }^{20-21}$ in small organic molecules. Previous

7 studies ${ }^{22-23}$ suggested that LLPS occurs when the liquid-liquid phase boundary of the target

8 compound and solvent lie inside the metastable zone of the binary phase diagram. Taylor et al. ${ }^{23}$

9 have drawn some analogies between LLPS and colloidal formation, suggesting that the formation

10 of a DLP is the result of molecular aggregation, due to the inability to form energetically favourable

11 interactions. Similarly, our results suggest that the DLP, obtained by LLPS, is a consequence of a

12 drastic change of conditions when increasing amounts of acetone are added, resulting in a 4-

$13 \mathrm{APH}^{+} \mathrm{Cl}^{-}$self-assembly that promotes the formation of such complex phases, simultaneously

14 preventing the nucleation of the simpler monohydrate 3 . This would explain why the simple phase

153 was never obtained in the presence of acetone, even if the water content in the DLP was suitable

16 for the formation of 3 . This picture seems to recall some of the recent non-classical theories of

17 nucleation that describe the formation of a DLP, metastable with respect to the crystalline state, ${ }^{24-25}$

18 or the formation of pre-clusters ${ }^{26}$ prior to the ordering of a crystalline phase. To date, only few cases

19 with evidences of such mechanisms in small organic molecules have been reported. Previous work

20 on Ibuprofen, $^{27}$ glycine $^{28}$ and perylene diimides ${ }^{29}$ suggested non-classical mechanisms of

21 nucleation. However, the self-assembly and the crystal packing reported in our study have a

22 complexity not observed in the systems cited above. 
1 When we look at the different behaviours of this simple organic salt, we find it surprising that

2 fampridine hydrochloride can show such an extraordinary complexity. None of the factors which

3 might be associated with the formation of complex phases are present in phases $\mathbf{1}$ and $\mathbf{2}$, such as the

4 presence of metal centres ${ }^{30}$ able to promote specific coordination geometries or alkyl chains that

5 might induce micelle formation. ${ }^{11}$ Conversely, $4-\mathrm{APH}^{+} \mathrm{Cl}^{-}$is very small in size and its molecular

6 shape is relatively simple when compared to organic species usually studied in this context. ${ }^{7}$

7 Our results certainly represent a reality check for the crystal engineering community, suggesting 8 that predicting and controlling the rules governing supramolecular self-assemblies is still 9 challenging, even for simple molecules. However, we believe they will prompt further advances in this area and have an impact in several other directions. Phase $\mathbf{1}$ and $\mathbf{2}$ represent an unprecedented

11 discovery in the field of FK phases and the observation of spherical aggregates in the DLP might 12 open new perspectives in the field of supramolecular chemistry. We believe these objects, applied 13 in a supramolecular sense as building blocks and combined with other species, such as metals or 14 cavitands, might be used to design new classes of ionic liquids (e.g. porous liquids, luminescent or 15 magnetic ionic liquids) or crystalline materials (e.g. MOF and HOF). At the same time, they prompt 16 interesting questions: is it possible to further extend this family of FK phases suitably tuning the 17 content of water in the DLP, resulting in the isolation of other unknown hydrates? Moreover, can 18 other small molecules form such complex phases and what properties do the resulting materials have? We believe that answers to these questions will certainly provide the basis for the 20 development of new classes of materials.

\section{References}

1. Lehn, J.-M. Supramolecular chemistry—scope and perspectives molecules, supermolecules, and molecular devices (nobel lecture). Angew. Chem. Int. Ed. Engl. 27, 89-112 (1988). 
2. Whitesides G. M., Mathias J. P., Seto C.T. Molecular self-assembly and nanochemistry: a chemical strategy for the synthesis of nanostructures. Science. 254, 1312-1319, (1991)

3. Lehn, J.-M. Perspectives in supramolecular chemistry-from molecular recognition towards molecular information processing and self-organization. Angew. Chem. Int. Ed. Engl. 29, 1304-1319 (1990).

4. Frank, F. C. \& Kasper, J. S. Complex alloy structures regarded as sphere packings. I. Definitions and basic principles. Acta Crystallograph. 11, 184-190 (1958).

5. Frank, F. C. \& Kasper, J. S. Complex alloy structures regarded as sphere packings. II. Analysis and classification of representative structures. Acta Crystallograph. 12, 483-499 (1959).

6. Ungar, G. \& Zeng, X. Frank-Kasper, quasicrystalline and related phases in liquid crystals. Soft Matter 1, 95-106 (2005).

7. Huang, M. et al. Selective assemblies of giant tetrahedra via precisely controlled positional interactions. Science 348, 424-428 (2015).

8. Zhang, J. \& Bates, F. S. Dodecagonal quasicrystalline morphology in a poly(styrene-bisoprene-b-styrene-b-ethylene oxide) tetrablock terpolymer. J. Am. Chem. Soc. 134, 76367639 (2012).

9. Reddy, A. et al. Stable Frank-Kasper phases of self-assembled, soft matter spheres. Proc. Natl. Acad. Sci. U. S. A. 115, 10233-10238 (2018).

10. Lee, S., Bluemle, M. J. \& Bates, F. S. Discovery of a Frank-Kasper $\sigma$ phase in sphereforming block copolymer melts. Science 330, 349-353 (2010).

11. Kim, S. A., Jeong, K.-J., Yethiraj, A. \& Mahanthappa, M. K. Low-symmetry sphere packings of simple surfactant micelles induced by ionic sphericity. Proc. Natl. Acad. Sci. U. S. A. 114, 4072-4077 (2017).

12. Goodfellow, B. W. et al. Ordered structure rearrangements in heated gold nanocrystal superlattices. Nano Lett. 13, 5710-5714 (2013).

13. Su, Z., et al. Identification of a Frank-Kasper Z phase from shape amphiphile selfassembly. Nat. Chem. 11, 899-905 (2019).

14. Macksasitorn, S. Hu Y. \& Stork J. R. Homoconjugated 4-aminopyridine salts: influence of anions on network topology CrystEngComm 15, 1698-1705, (2013).

15. Montis, R. \& Hursthouse, M. B. Crystalline adducts of some substituted salicylic acids with 4-aminopyridine, including hydrates and solvates: contact and separated ionic complexes with diverse supramolecular synthons. CrystEngComm 14, 7466-7478 (2012).

16. Hursthouse, M. B. et al. Anhydrates and/or hydrates in nitrate, sulphate and phosphate salts of 4-aminopyridine, (4-ap) and 3,4-diaminopyridine (3,4-dap): the role of the water molecules in the hydrates. CrystEngComm 16, 2205-2219 (2014). 
17. Kukkonen E., Malinen H. Haukka M., Konu J. Reactivity of 4-Aminopyridine with Halogens and Interhalogens: Weak Interactions Supported Networks of 4-Aminopyridine and 4-Aminopyridinium Cryst.Growth Des., 19, 4, 2434-2445 (2019).

18. Nguyen, A. H. \& Molinero, V. Stability and metastability of bromine clathrate polymorphs. J. Phys. Chem. B 117, 6330-6338 (2013).

19. Taratuta, V. G., Holschbach, A., Thurston G. M., Blankschtein, D., Benedek, G. B. Liquid-Liquid Phase Separation of Aqueous Lysozyme Solutions: Effects of $\mathrm{pH}$ and Salt Identity J. Phys. Chem., 94, 5, 2140-2144 (1990).

20. Deneau, E. \& Steele, G. An in-line study of oiling out and crystallization. Org. Process Res. Dev. 9, 943-950, (2005).

21. Veesler, S., Revalor, E., Bottini, O. and Hoff, C. Crystallization in the Presence of a Liquid-Liquid Phase Separation. Org. Process Res. Dev. 10, 841-845, (2006).

22. Bonnett P. E., Carpenter K. J. Dawson S. and Davey R. Solution crystallisation via a submerged liquid-liquid phase boundary: oiling out. Chem.Comm. 698-699 (2003)

23. Ilevbare G. A. and Taylor L. S. Liquid-Liquid Phase Separation in Highly Supersaturated Aqueous Solutions of Poorly Water-Soluble Drugs: Implications for Solubility Enhancing Formulations. Cryst. Growth Des. 13, 4, 1497-1509 (2013).

24. ten Wolde, P. R., Frenkel, D. Enhancement of Protein Crystal Nucleation by Critical Density Fluctuations. Science, 277, 1975-1978 (1997).

25. Pan, W. Kolomeisky, A. B. Vekilov, P. G. Nucleation of ordered solid phases of proteins via a disordered high-density state: Phenomenological approach. J. Chem. Phys., 122, 174905 (2005).

26. Gebauer, D. Volkel, A. Cölfen, H. Stable Prenucleation Calcium Carbonate Clusters. Science, 322, 1819- 1822 (2008).

27. Wiedenbeck, E., Kovermann, M., Gebauer, D., and Colfen, H., Liquid Metastable Precursors of Ibuprofen as Aqueous Nucleation Intermediates. Angew. Chem. Int. Ed. 58, 2-9 (2019).

28. Zaccaro, J., Matic, J., Myerson A.S., and Garetz, B.A., Nonphotochemical, laser-induced nucleation of supersaturated aqueous glycine produces unexpected $\gamma$-polymorph." Cryst. Growth Des. 1, 1, 5-8 (2001).

29. Tsarfati, Y. et al. Crystallization of Organic Molecules: Nonclassical Mechanism Revealed by Direct Imaging. ACS Centr. Sci. 4,8, 1031-1036 (2018).

30. Ecija, D. et al. Five-vertex Archimedean surface tessellation bylanthanide-directed molecular self-assembly. Proc. Natl. Acad. Sci. U. S. A. 110, 6678-6681 (2013). 
Figure 1. Crystal packing and self-assembly of phase 1 (a) and phase 2 (b). Clusters A1, A2, $B$ and $C$ are coloured in purple, orange, blue and grey respectively. In both structures, disorder has been removed for clarity. Chlorides have been connected to highlight the 4$\mathrm{APH}^{+} \mathrm{Cl}^{-}$polyhedral self-assembly.

Figure 2. Self - assembly in solid-state. (a) Hexagonal and pentagonal assemblies; (b) Clusters A1, A2, B and C; (c) chains of A clusters; (d) Instance of interstitial clusters C within the coordination of A1 A2 and $B$, illustrated by the of CN12 case; (e) coordination CN12 and CN14 for A1 A2 and B, shown in details (top) and as schematic representation (bottom) viewed along the directions $c, a b$ and $a b$, respectively. (f) Schematic representation of the FK phase A15. Colour coding: purple (cluster A1), orange (cluster A2), blue (cluster B) and grey (cluster $\mathrm{C}$ ). Chlorides have been connected to highlight the 4-APH ${ }^{+} \mathrm{Cl}^{-}$ polyhedral self-assembly.

Figure 3. Self - assembly in liquid-state. (a) Liquid-state ${ }^{1} \mathrm{H} \mathrm{NMR}$ spectra of 4-APH${ }^{+} \mathrm{Cl}^{-}$and (b) ${ }^{35} \mathrm{CI}$ NMR spectra of 4-APH ${ }^{+} \mathrm{Cl}^{-}$showed for $\mathrm{L1}$ (black) and $\mathrm{L3}$ (red) and collected for both liquids at a molar fraction of 4- $\mathrm{APH}^{+} \mathrm{Cl}^{-}$of 0.13 . Variation of the chemical shift of (c) the ${ }^{1} \mathrm{H}$ signal of the $\mathrm{NH}$ group and (d) ${ }^{35} \mathrm{Cl}$ signal as the function of the 4- $\mathrm{APH}^{+} \mathrm{Cl}^{-}$molar fraction, showed for L1 (black) and L3 (red). (e) Variation of full linewidth at the half height of the ${ }^{35} \mathrm{Cl}$ signal as a function of the 4-APH${ }^{+} \mathrm{Cl}^{-}$molar fraction, showed for $\mathrm{L1}$ (black) and $\mathrm{L3}$ (red). (f) Cryo-EM images of objects embedded in the frozen DLP sample obtained in presence of acetone, after the LLPS. Three zones (1-3) containing the objects are magnified and reported in panel 1-3. The bottom row shows the same zones after a further image processing (FFT bandpass filter, filtering features smaller than $5 \AA$, followed by a further autoscaling of contrast and brightness) to reduce the images low $\mathrm{S} / \mathrm{N}$ ratio and enhance the contrast of the imaged objects. The scale bar reported in each panel corresponds to a length of $10 \mathbf{~ n m}$.

Methods 
4-AP 98\% (4-AP) starting material was purchased from ACROS ORGANICS, hydrochloric acid 37\% ( $\mathrm{HCl})$ was purchased from Fisher Scientific. Solvents were all analytical grade. All reagents were used without further purification.

\section{Preparation of 1-4}

Phase 1: 4-aminopyridinium chloride 1/12 $\mathrm{H}_{2} \mathrm{O}$. Single crystals of phase 1 were serendipitously obtained during a solvent evaporation using a rotary evaporator. The preparation was made as follows. To a solution of 4-AP (4 g, $42 \mathrm{mmol}$ ) in $1.5 \mathrm{~mL}$ of ethanol was added dropwise an equimolar amount of concentrated $\mathrm{HCl}(3.5 \mathrm{~mL}, 42 \mathrm{mmol})$. The resulting solution was reduced in volume at low pressure and approximately 55-60 ${ }^{\circ} \mathrm{C}$ using a rotary evaporator, resulting in a dense liquid. In order to facilitate a further evaporation of water, an excess of ethanol was added to the liquid to promote the formation of the azeotrope water/ethanol. After further evaporation, the dense liquid was cooled, producing crystals of phase $\mathbf{1}$ suitable for single-crystal X-ray diffraction.

In general, phase $\mathbf{1}$ can be reproducibly crystallised by solvent diffusion or vapour diffusion from concentrated solutions of $4-\mathrm{APH}^{+} \mathrm{Cl}^{-}$in methanol or ethanol, using acetone as the antisolvent. Extended data 1 shows a typical crystallisation experiment, in which details of the liquid-liquid phase separation (LLPS) prior the crystallisation of phase $\mathbf{1}$ have also been reported. Starting from a solution of $4-\mathrm{APH}^{+} \mathrm{Cl}^{-}$in ethanol, obtained by mixing $1.0 \mathrm{~g}$ of 4-AP (10.6 mmol) in $2.5 \mathrm{~mL}$ of ethanol and adding dropwise $1.0 \mathrm{~mL}$ of $\mathrm{HCl} 37 \%$ (12.0 mmol), aliquots of acetone have been added to promote the precipitation of phase 1 (Extended Data 1a). After each addition of acetone, the solution became initially cloudy, suggesting a liquid-liquid demixing. After stirring, this immediately turned to a transparent isotropic liquid. When approximately $9 \mathrm{~mL}$ of acetone were added, the solution showed persistent cloudiness, even after vigorous stirring (red point in Extended Data 1a). Droplets of the separated liquid (Extended Data 1b) decanted in 50 minutes, 
1 forming a DLP at the bottom of the vial (Extended Data 1c). Adding further amounts of acetone

2 promotes a further separation of the DLP. Upon addition of approximately $70 \mathrm{~mL}$ of acetone the

3 DLP solidifies, resulting in colourless crystals of phase 1 (Extended Data 1d).

4 A slightly different method consisted of bubbling gaseous $\mathrm{HCl}$ into a solution of 4-AP in mixtures

5 of ethanol or $\mathrm{MeOH}$ and acetone. The formation of the salt in the solution containing an excess of

6 acetone resulted in the precipitation of phase $\mathbf{1}$. Although macroscopic LLPS were not observed

7 during this preparation, droplets formation similar to those showed in Extended Data 1 prior the

8 precipitation of phase 1 cannot be excluded.

9 Phase 2: 4-aminopyridinium chloride $1 / 90 \mathbf{H}_{2} \mathrm{O}$. Single crystals of phase 2 suitable for X-ray 10 investigations (Extended Data 3b) were serendipitously obtained during an attempt to recrystallise

11 phase 4 from the melt. Crystals of phase 4 that were left under the atmosphere of the lab for 12 approximately 30 minutes, were heated up until melting and recrystallized by slow cooling from 13 the melt, resulting in block-like colourless crystals of phase 2. In some experiments, crystals of 14 phase $\mathbf{2}$ were also obtained concomitantly during the preparation of phase $\mathbf{1}$ from the DLP.

15 Phase 3: 4-aminopyridinium chloride monohydrate. Single crystals of phase $\mathbf{3}$ were 16 reproducibly obtained by solvent evaporation from aqueous solutions of the 4- $\mathrm{APH}^{+} \mathrm{Cl}^{-}$salt.

17 Phase 4: 4-aminopyridinium chloride. Crystals of phase 4 (Extended Data 3d) were obtained by 18 recrystallising phase $\mathbf{1}$ by slow cooling from the melt, in the temperature range of $170-160{ }^{\circ} \mathrm{C}$.

\section{Single-Crystal X-ray Diffraction.}

20 X-ray diffraction data for phases $\mathbf{1}$ and $\mathbf{3}$ were collected at a temperature of 120(2) K using a 21 Bruker-Nonius APEX II diffractometer situated at the window of an FR591 rotating anode (MoKa, $22 \lambda=0.71073 \AA$ ). Data for phases 2 and 4 were collected at a temperature of 100(2) K using a Rigaku $23 \mathrm{FRE}+(\mathrm{MoKa}, \lambda=0.710735 \AA)$ rotating anode equipped with VHF Varimax confocal mirrors, and 
an AFC12 goniometer and a HyPix 6000 detector. The structures were solved and refined using

2 the SHELX suite of programs ${ }^{31-33}$ and hydrogen atoms were located in calculated positions with

3 the thermal parameter riding on the value of the parent atom. In order to make a preliminary

4 examination of the apparent relationships between the occupancies of the disordered components,

5 a further constrained refinement of phase 1 was made with idealized, fixed n/6 occupancies. The

6 resulting $\mathrm{R}$ value was very close to that of the first refinement (see Extended Data 2).

7 Figures 1 and 2 were prepared using the software Mercury CSD 3.10.3 and VMD 1.9.3 software.

8 Primary crystallographic data collection and refinement parameters are shown in Extended Data

$9 \quad 2$, further details on phases $\mathbf{1 - 4}$ are reported in Supplementary Information.

\section{Self-Assembly}

11 Extended Data 5 shows the main self-assemblies observed in phases 1-4. Phases 1, 2 and 4 show common hexagonal and pentagonal self-assemblies of 4- $\mathrm{APH}^{+}$and $\mathrm{Cl}^{-}$. Each hexagon or pentagon assembly, is formed by 4- $\mathrm{APH}^{+}$cations surrounded by $\mathrm{Cl}^{-}$anions and interacting by $\mathrm{N}-\mathrm{H} . . . \mathrm{Cl}^{-}$and

$14 \mathrm{C}-\mathrm{H} . . . \mathrm{Cl}^{-}$hydrogen bonds, with $\mathrm{N}_{\text {... }} \mathrm{Cl}^{-}$and $\mathrm{C} . . . \mathrm{Cl}^{-}$distances in the range 3.0-3.3 $\AA$ and 3.2-3.5

$15 \AA$ respectively. In the case of phases $\mathbf{1}$ and 2, the hexagons and pentagons are assembled to form 16 polyhedral clusters (see main text and Extended Data 5), while in the case of phase 4 these are 17 arranged in 2-D planes (see Extended Date 5 and Supplementary Information 1.4). In case of the 18 polyhedra, pentagonal and hexagonal tiles are capped by a further $\mathrm{Cl}^{-}$anion that in some cases 19 interacts with the 4- $\mathrm{APH}^{+}$via $\pi . . . \mathrm{Cl}^{-}$interactions $\left(\mathrm{Cl}^{-} \ldots\right.$ centroid distance in the range 3.4-3.7 $\AA$ ).

20 Differently to phases $\mathbf{1}, 2$ and 4, phase 3 shows a self-assembly consisting of $\pi \ldots \pi$ stacking of 4-

$21 \mathrm{APH}^{+}$cations (centroids...centroids distances are in the range $3.60 \AA \hat{\AA}-3.76 \AA ̊$ ) and rhombus-like 22 tetramers of $\mathrm{Cl}^{-} \ldots \mathrm{H}-\mathrm{O}-\mathrm{H} \ldots \mathrm{Cl}-\left(\mathrm{Cl}^{-} \ldots \mathrm{H}\right.$ distances are in the range 2.26 Á- $2.51 \AA ̊$ ). 

6 clarity. follows.

\section{Packing of spheres.}

Crystal structures of phase $\mathbf{1}$ and phase $\mathbf{2}$ can be also interpreted as a packing of spheres. Each of the clusters A and B described in the main paper is indeed surrounded by instances of clusters C, resulting in fullerene-like polyhedra (see Extended Data 6 a-c). This set of spheres pack along the three dimensions, as shown in Extended Data 6 d-e, where 4- $\mathrm{APH}^{+}$and $\mathrm{Cl}^{-}$have been removed for

\section{Liquid-state NMR.}

${ }^{1} \mathrm{H},{ }^{13} \mathrm{C},{ }^{14} \mathrm{~N}$, and ${ }^{35} \mathrm{Cl} \mathrm{NMR}$ spectra were recorded lock-on, without sample spinning, on a spectrometer operating at $9.4 \mathrm{~T}\left(400.0,100.5,28.8\right.$, and $39.2 \mathrm{MHz}$, respectively) and $25^{\circ} \mathrm{C}$, equipped with a $5 \mathrm{~mm}$ broadband probe and temperature regulation. Quantitative ${ }^{1} \mathrm{H}$ NMR spectra were recorded using the following acquisition parameters: relaxation delay of $15.0 \mathrm{~s}$, acquisition time of $5.0 \mathrm{~s}$, excitation pulse of $9^{\circ}$, and 32 transients. ${ }^{14} \mathrm{~N}$ and ${ }^{35} \mathrm{Cl} \mathrm{NMR}$ spectra were recorded using the Ring Down Elimination (RIDE) pulse sequence to reduce distortion of the baseline.

The ${ }^{1} \mathrm{H}$ and ${ }^{13} \mathrm{C}$ chemical shifts were referenced to the methyl signal of 3-(Trimethylsilyl)-1propanesulfonic acid sodium salt (DSS) which was used as internal reference. A cylindrical coaxial tube (Norell NI5CCI-V) with $\mathrm{NaCl} 1 \mathrm{M}$ in $\mathrm{D}_{2} \mathrm{O}$ was used for the ${ }^{2} \mathrm{H}$ lock and as external ${ }^{35} \mathrm{Cl}$ chemical shift reference (0.0 ppm). Preparation of the solutions $\mathbf{L} \mathbf{1}$ and $\mathbf{L} 3$ was carried out as

L1. Crystallisation of phase $\mathbf{1}$ was characterised by NMR. The sample was prepared by dissolving $1.0 \mathrm{~g}$ of 4 -AP $(10.6 \mathrm{mmol})$ in $2.5 \mathrm{~mL}$ of ethanol and adding dropwise $1.0 \mathrm{~mL}$ of $\mathrm{HCl} 37 \%$ (12.0 mmol). Upon addition of $9 \mathrm{~mL}$ of acetone, a persistent LLPS was observed. The composition of the DLP was then analysed upon addition of further amount of acetone, until the moment the crystallisation of phase 1 was observed. 
1 L3. An aqueous $4-\mathrm{APH}^{+} \mathrm{Cl}^{-}$solution for the characterisation of crystallisation of phase 3 was

2 prepared by dissolving $1.0 \mathrm{~g}$ of $4-\mathrm{AP}(10.6 \mathrm{mmol})$ in around $65 \mathrm{~mL}$ of $\mathrm{H}_{2} \mathrm{O}$ and $1.0 \mathrm{~mL}$ of $\mathrm{HCl}$

$337 \%$ (12.0 mmol). $500 \mu \mathrm{L}$ of the solution were transferred in a $5 \mathrm{~mm}$ NMR tube for the NMR

4 characterisation. Then, the solution was dried under reduced pressure using a rotavapor and

5 analysed again by NMR. The described procedure was repeated several times until the

6 crystallisation of phase $\mathbf{3}$ was observed.

\section{6. Cryo-EM}

8 The Cryo-EM imaging was performed by depositing a droplet of the sample (2 $\square$ l) on copper 200

9 mesh grids covered with a Quantifoil R2/2 holey carbon film at $24{ }^{\circ} \mathrm{C}$, under room pressure and

10 in air, being the sample slightly hygroscopic. Then, keeping fixed the temperature, the grid was

11 blotted with filter paper to obtain a very thin layer of liquid sample suspended in every hole of the

12 holey grid, by using a FEI Vitrobot Mark IV, with blotting force 2 and for a blotting time of 2.5 s.

13 Both these parameters were carefully chosen in order to most properly get the thin amorphous

14 layer of the sample vitrified onto the grid, and basically depended on both viscosity and volatility

15 of the sample, that were revealed quite different from that of a solution constituted by water as a

16 solvent. The small amount of sample liquid films kept on the grid after the blotting procedure was

17 then frozen in an amorphous state by fast plunging it in liquid ethane kept at $-175{ }^{\circ} \mathrm{C}$, where the sample was previously checked to be totally immiscible. Then the frozen grid was transferred into

19 liquid nitrogen and mounted on the samples’ container of a FEI Titan Krios transmission electron

20 microscope, equipped with a Schottky source, and a cryo-twin objective lens. The Cryo-EM

21 imaging was performed exploiting the phase contrast produced by the imaged objects, with the

22 microscope operating at an acceleration voltage of $300 \mathrm{kV}$. The images were acquired by a

23 dedicated Gatan Image Filter Model (GIF) Model 968, retrofitted with an ultrasensitive Gatan K2 
1 Summit CMOS camera, collecting only the electrons with no loss in energy (zero-loss peak (ZLP)

2 images) upon low electron dose conditions $\left(\sim 10 \mathrm{e} / \AA^{2}\right)$ to enhance the sample images contrast, and

3 with a defocus value of $-2000 \mathrm{~nm}$, the latter retrospectively determined by the CTFFIND4

$4 \operatorname{program}^{34}$. Prior to vitrification, the system was exposed to the laboratory atmosphere $\left(20-25{ }^{\circ} \mathrm{C}\right.$,

51 atm and approximately 40\%RH) for less than 5 seconds (from the drop deposition on the TEM

6 grid to the immersion of the film into the cryogen). In particular, less than 0.5 seconds passed from

7 the end of the blotting phase and the grid immersion into the liquid ethane. We estimated during

8 the latter time a partial evaporation of acetone resulting in a decrease of the vitrified layer thickness

9 to approximately $80-100 \mathrm{~nm}$ from the initial $100-130$ nm-thick film.

10 Analysis of the DLP by cryo-EM revealed the presence of objects with size ranging between 1.5

11 and $4.6( \pm 0.5) \mathrm{nm}$. A selection of these objects is reported in Figure 3 (see main article). Extended

12 Data 7 shows objects with size in the range 1.5- $2.8( \pm 0.5) \mathrm{nm}$.

13 Data Availability Statement. Full crystallographic details in CIF format have been deposited in

14 the Cambridge Crystallographic Data Centre (Deposition number: Phase $\mathbf{1}=$ CCDC 1540139

15 (unconstrained) and 1540140 (constrained), Phase 2 = CCDC 1897427, Phase 3 = CCDC 1540141,

16 Phase 4 = 1897428). Copies of this information may be obtained free of charge from the Director,

17 CCDC, 12 Union Road, Cambridge, CB2 1EZ (fax +44 1223 336033) or email:

18 deposit@ccdc.cam.ac.uk or www: http://www.ccdc.cam.ac.uk. Raw single-crystal diffraction data

19 corresponding to the structures of Phases 1-4 have been deposited in the Zenodo repository at the

20 following locations: Phase 1 https://doi.org/10.5281/zenodo.2595089; Phase

212 https://doi.org/10.5281/zenodo.2585776; Phase 3 https://doi.org/10.5281/zenodo.2593670;

22 Phase 4 https://doi.org/10.5281/zenodo.2593677. The CCD images (in either Rigaku IMG or

23 Bruker KCD format) have been deposited, along with instrument parameters and all files 
associated with image processing. This will enable the reader to fully validate these structural models and for those who wish to investigate alternative approaches to modelling these extraordinary results or develop them further it will be possible to do so without having to synthesise the materials and collect diffraction data. NMR results are extensively described in Supplementary Information (section S5), data are available on request. A selection of relevant cryo-EM images is included in the manuscript (Figure 3 and Extended Data 7). Further data are available on request.

31. Sheldrick, G. M. Crystal structure refinement with SHELXL. Acta Crystallogr., Sect. C: Struct. Chem. 71, 3-8 (2015).

32. Sheldrick, G. M. SHELXT - Integrated space-group and crystal-structure determination. Acta Crystallogr., Sect. A: Foundations and Advances. 71, 3-8 (2015).

33. Sheldrick, G. M. Crystal structure refinement with SHELXL. Acta Crystallogr., Sect. C: Struct. Chem. 71, 3-8 (2015).

34. Rohou, A. Grigorieff N., CTFFIND4: fast and accurate defocus estimation from electron micrographs. J. Struc. Biol. 192, 216-221 (2015).

\section{Acknowledgements}

The authors would like to thank the UK Engineering and Physical Sciences Research Council for financial support for single-crystal diffraction facilities through funding the UK National Crystallography Service. RM thanks Prof. Roger Davey (The University of Manchester) for valuable comments and discussions. Dr. Morgane Sanselme (Université de Rouen Normandie) is thanked for help in in-situ X-ray diffraction measurements. The authors thank theTechnological Platform "Physico-Chemical Characterization” - PC (University of Namur) for providing resources used for this research.

\section{Author Contributions}


R. M. defined the protocol for crystallisations of phases 1-4 and performed the initial preparations and crystallisations. R. M., M. B. H. and P. N. H. performed the single-crystal data collections and crystal structure refinements. R. M. and A. D. R. analysed the crystal structures. R. M. and L. F. described structures $\mathbf{1}$ and $\mathbf{2}$ as Frank-Kasper phases and produced all the relevant images.

L. F. performed the NMR characterisation and independently carried out reproducibility crystallisation experiments. N. T. characterised by PXRD phases 1 - 4. G. C. and A. L. independently performed reproducibility crystallisation experiments and PXRD characterisation of phases $\mathbf{1}$ and $\mathbf{3}$ and conducted the humidity measurements. Thermal Analysis was performed independently by University of Namur (N.T. and L.F.), Université de Rouen Normandie (G. C. and A.L.) and University of Southampton (R. M. and P. N. H.) A. F. and R.S. performed the cryo-EM measurements and the analysis of the results.

R. M., L. F., A. D. R., T. L. T., M. B. H., N. T. and S. J. C. undertook extensive analysis of the results and wrote the manuscript.

\section{Additional Information}

Supplementary Information is available for this paper.

Competing financial interests: The authors declare no competing financial interests.

Materials \& Correspondence. Correspondence and requests for materials should be addressed to R.M. (riccardo.montis@gmail.com).

Extended Data 1. LLPS in 4-APH ${ }^{+} \mathrm{Cl}^{-}$. (a) Starting solution and LLPS upon addition of various amount of acetone; (b) droplets formation upon addition of acetone; (c) evolution of the droplets formation as the function of the time and separation of the DLP at the bottom of the vial; (d) comparison of the starting solution, after separation of droplets and 
crystals separated from the DLP. Sample showing a persistent cloudiness is indicated with a red circle.

Extended Data 2. Crystal data for phase 1-4

Extended Data 3. Examples of crystals of phases 1-4: (a) phase 1, (b) phase 2, (c) phase 3, (d) phase 4. Crystals for phase 2 and 4 have been obtained by slow cooling crystallisation from the melt of phase 1 .

Extended Data 4. Crystal packing of Phases 1-4, viewed along the three axes of the unit cell. (a) Phase 1; (b) phase 2; (c) phase 3; (d) phase 4.

Extended Data 5. Main self-assemblies in phases 1-4. In the polyhedra, pentagonal and hexagonal tiles are capped by a further $\mathrm{Cl}$ - anion that in some cases interacts with the 4$\mathrm{APH}^{+}$via $\pi . . . \mathrm{Cl}^{-}$interactions $\left(\mathrm{Cl}^{-}\right.$...centroid distance in the range $\left.3.4-3.7 \AA\right)$.

Extended Data 6. Fullerene-like polyhedra around (a) clusters A1, (b) A2 and (c) B shown for phase 1 as representative. Packing of fullerene-like spheres. (d) Phase 1; (e) Phase 2.

Extended Data 7. Cryo-EM images of small objects embedded in the frozen DLP sample obtained the LLPS promoted by the antisolvent acetone One zone per image containing the small objects is magnified and reported in bottom panels 1 and 2, which show the same zones after a further image processing (FFT bandpass filter, filtering features smaller than $5 \AA$, followed by a further autoscaling of contrast and brightness) to reduce the images low $\mathrm{S} / \mathrm{N}$ ratio and enhance the contrast of the imaged objects. In panel 1 the black arrow indicates a spherical object with diameter of $1.5 \pm 0.5 \mathrm{~nm}$, while in panel 2 it indicates a further, isolated spherical object with diameter of $2.8 \pm 0.5 \mathrm{~nm}$. The scale bar reported in the bottom panels corresponds to a length of $10 \mathbf{~ n m}$. 